\title{
Analytical Strategy for the Molecular Weight Determination of Random Copolymers of Poly(Methyl Methacrylate) and Poly(Methacrylic Acid)
}

\author{
Rémi Giordanengo, ${ }^{a}$ Stéphane Viel, ${ }^{\mathrm{b}}$ Manuel Hidalgo, ${ }^{\mathrm{c}}$ \\ Béatrice Allard-Breton, ${ }^{\mathrm{c}}$ André Thévand, ${ }^{\mathrm{a}}$ and Laurence Charles ${ }^{\mathrm{a}}$ \\ ${ }^{a}$ Universités Aix-Marseille I, II, and III-CNRS, UMR 6264: Laboratoire Chimie Provence, Spectrométries \\ Appliquées à la Chimie Structurale, Marseille, France \\ b Aix-Marseille Université-CNRS, UMR 6263: Institut des Sciences Moléculaires de Marseille, Chimiométrie \\ et Spectrométries, Marseille, France \\ c ARKEMA, Centre de Recherche Rhône Alpes, Pierre-Bénite, France
}

\begin{abstract}
Molecular weight characterization of random amphiphilic copolymers currently represents an analytical challenge. In particular, molecules composed of methacrylic acid (MAA) and methyl methacrylate (MMA) as the repeat units raise issues in commonly used techniques. The present study shows that when random copolymers cannot be properly ionized by MALDI, and hence detected and measured in MS, one possible analytical strategy is to transform them into homopolymers, which are more amenable to this ionization technique. Then, by combining the molecular weight of the so-obtained homopolymers, as measured by MS, with the relative molar proportion of the MMA and MMA units, as given by ${ }^{1} \mathrm{H}$ NMR spectrum, one can straightforwardly estimate the molecular weight of the initial copolymer. A methylation reaction was performed to transform MAA-MMA copolymer samples into PMMA homopolymers, using trimethylsilyldiazomethane as a derivatization agent. Weight average molecular weight $\left(M_{\mathrm{w}}\right)$ parameters of the MAA-MMA copolymers could then be derived from $M_{\mathrm{w}}$ values obtained for the methylated MAA-MMA molecules by MALDI, which were also validated by pulsed gradient spin echo (PGSE) NMR. An alkene function in one of the studied copolymer end-groups was also shown to react with the methylation agent, giving rise to MMA-like polymeric by-products characterized by tandem mass spectrometry and which could be avoided by adjusting the amount of the trimethylsilyldiazomethane in the reaction medium. (J Am Soc Mass Spectrom 2010, 21, 1075-1085) @ 2010 American Society for Mass Spectrometry
\end{abstract}

$\mathrm{P}$ olymers based on weak acids such as poly(methacrylic acid) (PMAA) have attracted considerable attention because of the ability of the system to change strongly upon variations in the $\mathrm{pH}$ and ionic strength of the solution [1]. For example, amphiphilic PMAA-based block copolymers were reported to efficiently adsorb uranyl ions [2] or to selfassemble to produce dynamic micelles sensitive to different stimuli [3-6]. In particular, block copolymers containing PMAA and poly(methyl methacrylate) (PMMA) segments were the subject of intensive research activities [7-10]. However, interesting properties were also demonstrated for copolymers containing random MAA-MMA segments [11-14]. Performance of

Address reprint requests to Dr. L. Charles, Universités Aix-Marseille I, II \& III, Laboratoire Chimie Provence, Spectrométries Appliquées à la Chimie Structurale, Campus Saint-Jérôme, Case 511, Avenue NormandieNiemen, 13397 Marseille Cedex 20, France. E-mail: laurence.charles@univprovence.fr such materials highly depends on structurally-related parameters but also on molecular weight distribution.

Determination of molecular weight parameters is not a trivial task for copolymers. Liquid state nuclear magnetic resonance (NMR) is the most commonly used technique for this purpose but only allows the number average molecular weight $\left(M_{n}\right)$ parameter to be obtained. Size exclusion chromatography (SEC) requires calibration with narrow standards of the same chemical nature and structure as the analyte. Alternatively, one can use calibration which relies on Mark-HouwinkKuhn-Sakurada (MHKS) parameters being known for the standards and the analyte [15], since these parameters depend on both the nature of co-monomers and their relative proportion within the copolymer [16]. As a result, a multiple detection configuration is usually required to reach reliable molecular weight data by SEC, since the intensity of the response obtained in each detection mode does not only depend on molecular weight but also on parameters that are a function of the 
macromolecule composition [16, 17]. Pulsed gradient spin echo (PGSE) [18-20] NMR can be used to estimate the weight average molecular weight $\left(M_{\mathrm{w}}\right)$ of a synthetic homopolymer from the measurement of its molecular self-diffusion coefficient [21-24]. For block copolymers, we have recently shown that such estimation not only requires properly recorded calibration curves for each of the polymers constituting the blocks, but also a hydrodynamic model to correctly interpret the diffusion data [25]. However, to the best of our knowledge, no such procedure has been reported so far to estimate $M_{\mathrm{w}}$ of random copolymers.

Mass spectrometry (MS) has become an increasingly important tool for polymer analysis since the development of soft ionization methods. As oligomers can be produced as intact molecular adducts using matrixassisted laser desorption/ionization (MALDI) [26, 27] or electrospray ionization (ESI) [26], MS spectra can be used to determine the masses of repeat units and end-groups, $M_{n}$ and $M_{\mathrm{w}}$ parameters, and thus polydispersity index (PDI), as long as polymer polydispersity is not too high. However, ESI of acrylic-based polymers usually gives rise to a strong mass bias toward low mass oligomers, as reported for both homopolymers [28-30] and copolymers [31]. Compared with ESI, MALDI was found to be more applicable to synthetic polymer analysis and lower mass biases were reported for acrylic-based homopolymers [32]. However, MALDI analysis of amphiphilic copolymers remains a challenge since each co-monomer requires its own set of experimental conditions for sample preparation. To circumvent this issue, we have recently developed a method consisting of hydrolyzing a targeted function in the junction group between the two segments of a block copolymer to produce two homopolymers which could then be independently mass-characterized using conventional MALDI protocols [33]. However, such a strategy can obviously be only envisaged for block copolymers.

The analytical methodology proposed here consists of the methylation of all MAA units to transform MAA-MMA copolymers into PMMA homopolymers, for which both MALDI and PGSE NMR experimental conditions are well established. Sample derivatization is a technique that has been widely used to enable gas chromatography analysis of small polar compounds. Methylation was also reported for PMAA homopolymers and allowed the accuracy of molecular weight parameters as determined by SEC to be improved [34], or signals obtained in MALDI to be enhanced and simplified [35]. This strategy has been successfully applied here to random MAA-MMA copolymers to enable the use of MALDI and PGSE for the determination of $M_{\mathrm{w}}$ parameters of the so-obtained PMMA homopolymers. Combining these data with monomer composition as determined by ${ }^{1} \mathrm{H}$ NMR allowed the $M_{\mathrm{w}}$ parameters of the studied copolymers to be determined.

\section{Experimental}

\section{Chemicals}

HPLC grade methanol and water were from SDS (Peypin, France) and used without further purification. $\mathrm{CD}_{3} \mathrm{OD}$ and deuterated acetonitrile $\left(\mathrm{ACN}-\mathrm{d}_{3}\right)$ used in NMR experiments were from Eurisotop (Saint-Aubin, France). Matrices used for MALDI sample preparation, 2,5-dihydroxybenzoic acid (2,5-DHB) (purity $\geq 98.5 \%$ ), sinapic acid (SA) (purity $\geq 99.0 \%$ ), and $2^{\prime}, 4^{\prime}, 6^{\prime}-$ trihydroxyacetophenone monohydrate (THAP) (purity $\geq$ $99.5 \%$ ), as well as $\mathrm{NaI}$ and $\mathrm{KI}$ salts used as cationizing agents, were purchased from Sigma-Aldrich (St. Louis, MO, USA). Poly(methacrylic acid) (PMAA) sodium salts with $M_{\mathrm{w}}$ 1250,1700, 3150, and $6600 \mathrm{~g} \mathrm{~mol}^{-1}$ were from PSS (Mainz, Germany). These PMAA standards have an $\mathrm{H}$ atom as both end-groups. Poly(ethylene glycol) (PEG) standards with $M_{\mathrm{w}} 1490,4270$, and $6650 \mathrm{~g}$ $\mathrm{mol}^{-1}$ used for mass analyzer calibration, as well as poly(methyl methacrylate) (PMMA) with $M_{\mathrm{w}}$ 1580, 1970, 4540, 7920, 40,300, and 48,200 $\mathrm{g} \mathrm{mol}^{-1}$ used for PGSE calibration, were from PSS. The two MAA-MMA copolymers (I and II) studied here, whose synthesis has been described in a previous paper [31], were kindly supplied by ARKEMA. Trimethylsilyldiazomethane (2 $M$ in diethyl ether) used as the methylation agent was from Sigma-Aldrich.

\section{Methylation Reaction}

The methylation procedure was derived from a published protocol [34] and is briefly described hereafter. MAA-MMA copolymers or PMAA homopolymers (50 $\mathrm{mg}$ ) were first dissolved in a methanol/water mixture $(19: 1, \mathrm{vol} / \mathrm{vol})$ and stirred at moderate speed for $2 \mathrm{~h}$. Trimethylsilyldiazomethane was then added dropwise until the polymer solution became yellow, indicating the methylation agent was in excess. The reaction mixture was then stirred for $3 \mathrm{~h}$ at ambient temperature and the so-obtained solution was evaporated to dryness overnight in an oven thermostated at $30^{\circ} \mathrm{C}$.

\section{Mass Spectrometry}

MALDI-TOF-MS experiments were carried out using a Bruker Autoflex I (Bruker Daltonics, Leipzig, Germany), equipped with a nitrogen laser emitting at 337 $\mathrm{nm}$, a single-stage pulsed ion extraction source and dual microchannel plate detectors. Data acquisition was performed in the reflectron mode, in positive or negative ion mode. The accelerating voltage was set to $19 \mathrm{kV}$ and the delay time used in delayed extraction mode (generally around $100 \mathrm{~ns}$ ) was optimized based on the mass range of the polymer distribution. FlexControl software version 2.2 (Bruker Daltonics) was used for instrument control and data acquisition, and FlexAnalysis software version 2.2 (Bruker Daltonics) for data processing. The MALDI mass spectra result from averages over 400 
consecutive laser shots at a $10 \mathrm{~Hz}$ frequency. All mass spectra were acquired at threshold laser irradiance. External calibration was performed using poly(ethylene glycol)s and poly(methyl methacrylate)s. PMAA homopolymers were dissolved in water while MAAMMA copolymers, methylated PMAAs and methylated copolymers were dissolved in methanol, at a $5 \mathrm{mg} \mathrm{mL}^{-1}$ concentration. The matrix $\left(10 \mathrm{mg} \mathrm{mL}^{-1}\right)$ and salt $(5 \mathrm{mg}$ $\mathrm{mL}^{-1}$ ) solutions were both prepared in methanol. SA and PMAA samples were mixed in a 1000:1 $\mathrm{M}$ ratio. THAP or 2,5-DHB were used for MALDI of methylated PMAA samples and the matrix/polymer molar ratios were optimized, based on the polymer size, in the 500:1-2000:1 range. THAP and NaI (or KI) were used to prepare the methylated MAA-MMA copolymer MALDI samples, with a matrix/salt/polymer ratio of 1000:10:1. All MALDI samples were prepared using the drieddroplet method, where $1 \mu \mathrm{L}$ of the liquid mixture was deposited onto the sample plate and allowed to air-dry.

High-resolution MS and MS/MS experiments were performed with a QStar Elite mass spectrometer (Applied Biosystems SCIEX, Concord, ON, Canada) equipped with an electrospray ionization source operated in the negative ion mode. The capillary voltage was set at $-4200 \mathrm{~V}$ and the cone voltage at $-70 \mathrm{~V}$. In this hybrid instrument, ions were measured using an orthogonal acceleration time-of-flight (oa-TOF) mass analyzer. A quadrupole was used for selection of precursor ions to be further submitted to collision-induced dissociations (CID) in MS/MS experiments. In MS, accurate mass measurements were performed using two reference ions from a poly(ethylene glycol) internal standard, according to a procedure described elsewhere [36]. The precursor ion was used as the reference for accurate measurements of product ions in the MS/MS spectra. Air was used as the nebulizing gas (10 psi) while nitrogen was used as the curtain gas (20 psi) and the collision gas. Analyst software version 2.1 (Applied Biosystems SCIEX) was used for instrument control, data acquisition and data processing. Methylated MAA-MMA copolymers were dissolved in methanol, further diluted to a final concentration of $50 \mu \mathrm{g} \mathrm{mL}^{-1}$ and introduced in the ionization source at a $5 \mu \mathrm{L} \mathrm{min}^{-1}$ flow rate using a syringe pump.

\section{Nuclear Magnetic Resonance}

${ }^{1} \mathrm{H}$ and PGSE experiments were performed on a Bruker Avance spectrometer operating at $500 \mathrm{MHz}$ for the ${ }^{1} \mathrm{H}$ Larmor frequency with a 5-mm triple resonance inverse Bruker cryoprobe optimized for ${ }^{1} \mathrm{H}$ detection and equipped with an actively shielded $z$-gradient coil. The gradient coil was calibrated by measuring the selfdiffusion coefficient of the residual proton in $\mathrm{D}_{2} \mathrm{O}$ and was found to be $55 \mathrm{G} \mathrm{cm}^{-1}$. The temperature was set and controlled to $300 \mathrm{~K}$ with an air flow of $545 \mathrm{~L} \mathrm{~h}^{-1}$ to avoid temperature fluctuations due to sample heating during the gradient pulses. Methylated PMAA and
MAA-MMA samples were prepared by weighing an amount of sample directly into the NMR tube and adding $0.6 \mathrm{~mL}$ of a mixture of methanol and acetonitrile deuterated solvents $(50 / 50, \mathrm{vol} / \mathrm{vol})$ to a final $3 \mathrm{mg}$ $\mathrm{mL}^{-1}$ concentration.

The diffusion of polymers is well understood and has been extensively studied [37]. The $M_{\mathrm{w}}$ parameter of a homopolymer can be related to its self-diffusion coefficient $D$, as measured in very dilute solutions, according to Flory's law [37] where scaling parameters that depend on the polymer structure as well as experimental conditions (solvent viscosity and temperature) are used. Typically, these scaling parameters are obtained by analyzing a series of monodisperse polymer standards under a given set of experimental conditions. The corresponding procedure has already been thoroughly described in other published works [24, 25] and will only be briefly described here. The PGSE diffusion decays are analyzed using a nonlinear least squared fitting (based on the Levenberg-Marquardt algorithm), which allows the diffusion coefficients to be obtained by taking the diffusion time and gradient pulse duration as input parameters. Then, by using a calibration curve that relates $D$ to $M_{\mathrm{w}}$, which is established from the analysis of polymers standards, the diffusion coefficients of the derivatized copolymers are subsequently used to determine their corresponding $M_{\mathrm{w}}$ values. The dependency on solvents is implicitly accounted for by using a calibration curve that is established in the same experimental conditions as those used to measure the $D$ values of the homopolymer standards. In this respect, a calibration curve was established by analyzing a set of PMMA homopolymer standards and was found to be such as $D=2.14010^{-8} M_{\mathrm{w}}{ }^{-0.4680}$.

\section{Results and Discussion}

Structural characterization of the two studied MAAMMA copolymers I and II was previously achieved combining information from MS and NMR analyses [31]. ${ }^{1} \mathrm{H}$ and ${ }^{13} \mathrm{C}$ NMR experiments revealed the structure of the $X$ and $Y$ end-groups (Scheme 1) as well as the proportion of each co-monomer in the molecules, found to be $40 / 60$ and 50/50 for copolymers I and II, respectively. The end-group masses were further confirmed from $\mathrm{m} / \mathrm{z}$ values of doubly charged copolymer anions detected in ESI-MS. However, copolymer composition derived from MS data were not consistent with NMR results, obviously due to strong mass biases well known to occur during electrospray ionization of these polymeric species. Tandem mass spectrometry revealed the random nature of the copolymer based on typical dissociation reactions: water elimination occurred from any two contiguous MAA units while MAAMMA pairs gave rise to the loss of a methanol molecule [31]. The $M_{n}$ parameter was derived from quantitative ${ }^{1} \mathrm{H}$ NMR experiments and was found to be 3400 and $4100 \mathrm{~g} \mathrm{~mol}^{-1}$ for MAA-MMA copolymers I and II, respectively. 


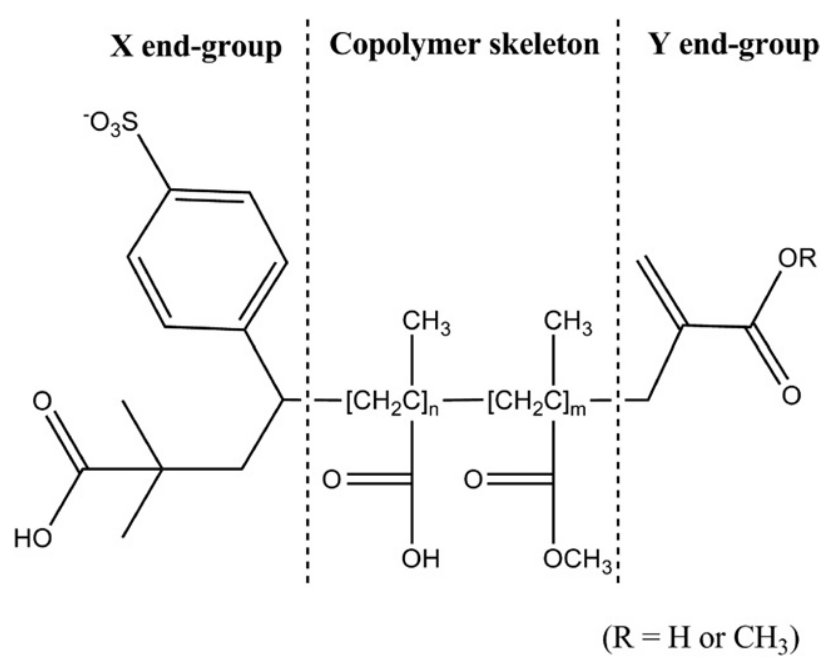

Scheme 1. Structure of the studied MAA-MMA copolymers.

The random copolymers were derivatized using trimethylsilyldiazomethane, to enable methylation of carboxylic acid groups, yielding a PMMA homopolymer. However, before examining random MAA-MMA copolymers, the derivatization procedure was first tested on PMAA homopolymers, with an $\mathrm{H}$ atom as both end-groups and various sizes in the 1200 to $6600 \mathrm{Da}$ mass range, using the protocol described by Couvreur et al. [34]. Before derivatization, PMAA homopolymers gave rise to complex mass spectra in negative mode MALDI (using sinapic acid as the matrix), as exemplified in Figure 1a for PMAA1700. The homopolymer was detected with low intensity as $\left[\mathrm{P}_{n}-\mathrm{H}\right]^{-}$ions, with $\mathrm{n}$ the polymerization degree, but also as deprotonated molecules with some sodiated carboxylic acid groups, $\left[\mathrm{P}_{n}+\mathrm{xNa}-(\mathrm{x}+1) \mathrm{H}\right]^{-}$ions (with $\mathrm{x}$ ranging from 1 to 3. Similar adducts were also formed from polymeric impurities, present in the studied standards, noted $\mathrm{P}_{n}^{\prime}$ and $\mathrm{P}_{n}{ }_{n}$ in the inset of Figure 1a, consisting of a PMAA homopolymer with respectively one and two MMA unit, as evidenced in previous studies [29, 30]. Mass spectra consisting of such multiple distributions, which have already been reported for poly(acrylic acid) or poly(styrene sulfonic acid) polymers [38], were obtained for all tested PMAA standards and a quite high laser fluence was usually required to generate the observed signals. After methylation of PMAA homopolymers, a unique PMMA distribution, consisting of sodiated adducts, $\left[\mathrm{Q}_{n}+\mathrm{Na}\right]^{+}$, and characterized by a $100 \mathrm{Da}$ mass difference between two consecutive oligomers, was obtained in positive mode MALDI (Figure 1b). In these experiments, 2,5-DHB was used as the matrix for MALDI of the smallest polymers $\left(M_{\mathrm{w}}<\right.$ $2000 \mathrm{~g} \mathrm{~mol}^{-1}$ ) while THAP was found to yield better quality mass spectra for the largest ones. It should be noted that MALDI-MS analysis of the derivatized samples in the negative ion mode did not show any residual PMAA distribution, indicating a $100 \%$ methylation yield for all tested standards. However, increasing volumes of trimethylsilyldiazomethane were required for methylation of increasing molecular weight PMAA, as indicated in Table 1, although the average number of carboxylic acid functions was the same (about 0.58 mmol). This result shows that the actual amount of derivatization agent to be used to ensure the methylation of all MAA monomers cannot be predicted and suggests different availability of the acidic groups as a function of the polymer size. The intense sodium adducts observed in Figure $1 \mathrm{~b}$ were formed from lower laser fluence, compared with ions in Figure 1a, and calculation of the end-group masses $\left(m_{X}+m_{Y}=2 \mathrm{Da}\right)$ confirmed all acidic functions of the original PMAA homopolymer have been methylated. Values obtained when using MALDI data to calculate $M_{\mathrm{w}}$ parameters for both PMAA and methylated-PMAA polymers are reported in Table 1 and were compared with SEC data provided by the supplier as well as $M_{\mathrm{w}}$ parameters obtained by PGSE NMR. The $M_{\mathrm{w}}{ }^{\text {MALDI }}$ values obtained for PMAA standards prior and after derivatization were consistent, with a maximal deviation of $12 \%$. Compared with SEC data provided by the supplier, a strong systematic bias towards low mass oligomers was observed for $M_{\mathrm{w}}$ values calculated from MALDI mass spectra of PMAA standards. However, molecular weights indicated by the supplier might not be used as reference values since they were derived from SEC calibration established using poly(t-butyl methacrylate) standards. These PMAA $M_{\mathrm{w}}$ SEC parameters could indeed be suspected to be overestimated since calculation, from the SEC data, of $M_{\mathrm{w}}$ expected for the methylated PMAA gave rise in most cases to higher values compared with $M_{\mathrm{w}}$ values obtained by both MALDI and PGSE NMR. In contrast, a good agreement was found between MALDI and PGSE data, although $M_{\mathrm{w}}$ MALDI appeared to be slightly underestimated when taking PGSE as the reference technique. Underestimation of $M_{\mathrm{w}}$ parameters is commonly reported for PMAA in both positive and negative mode MALDI [32]. However, relative deviations were quite low, with a maximal value of $15 \%$ for the smallest polymer and lying in the $4 \%-12 \%$ range for other samples, which is consistent with results obtained in a previous study [24].

The same methylation protocol was then applied to the MAA-MMA samples to transform these amphiphilic copolymers, for which no interpretable signal could be obtained in MALDI as illustrated in Figure 2a, into PMMA homopolymers more amenable to this ionization technique. Indeed, positive mode MALDI mass spectra exhibiting various polymeric distributions could readily be obtained for the methylated copolymers, using THAP and a sodium salt in the sample preparation (Figure $2 b$ ). Details of this mass spectrum show up to six PMMA distributions, which all experienced a +32 Da mass shift when changing the cationizing agent from $\mathrm{Na}^{+}$to $\mathrm{K}^{+}$, suggesting the substitution of two sodium by two potassium cations upon changing the salt in the MALDI sample preparation. This result indicates that one of the alkali cations must be 

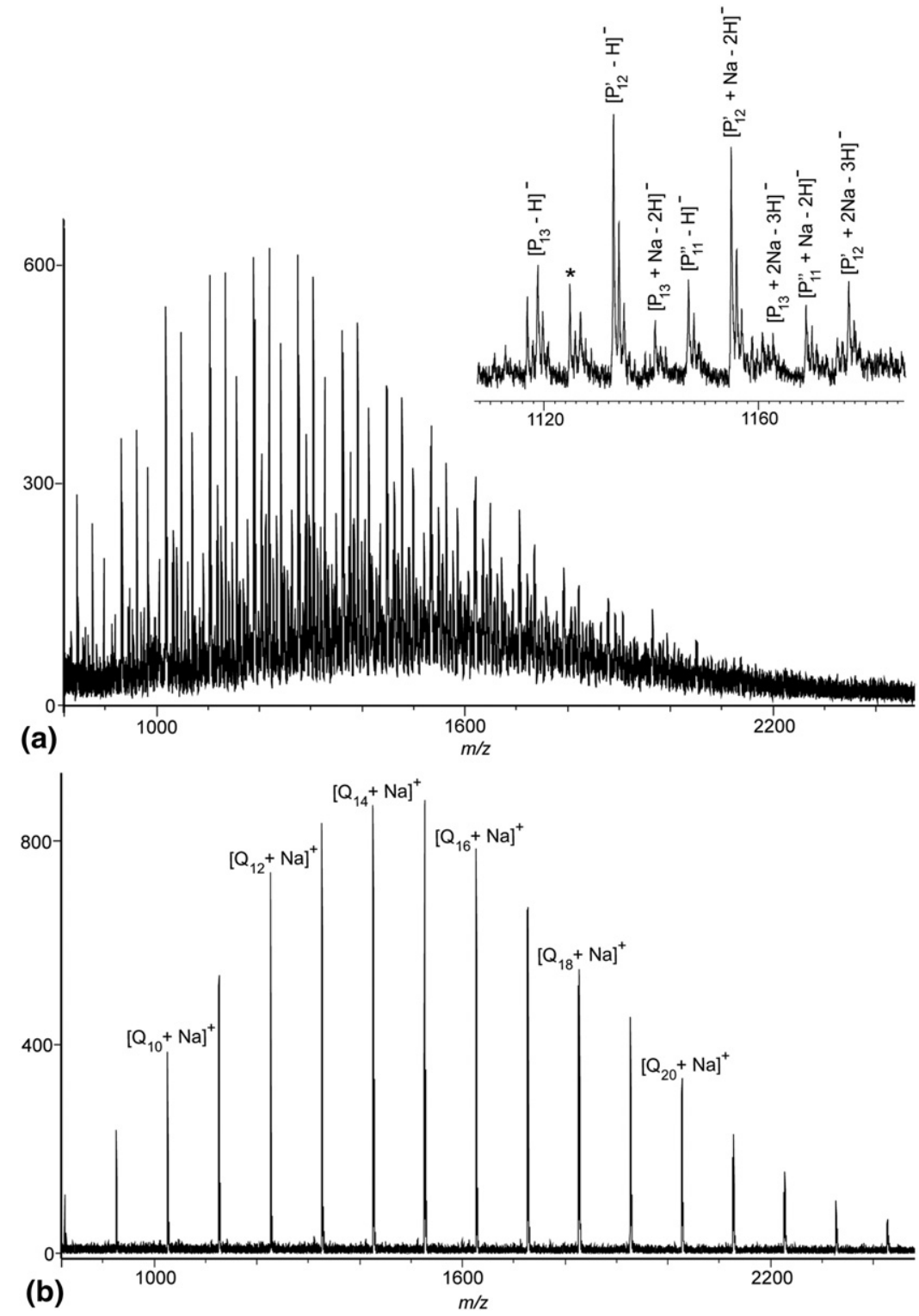

Figure 1. MALDI mass spectra obtained for (a) PMAA1700 in the negative mode, using SA as the matrix in a matrix-to-polymer ratio of 1000:1, and (b) methylated PMAA1700 in the positive mode, using 2,5-DHB as the matrix in a matrix-to-polymer ratio of 1000:1.

involved in a salt moiety within these singly charged oligomer adducts, which is consistent with the presence of a sulfonate function in the $X$ end-group of the studied MAA-MMA copolymers (Scheme 1). End-group mass calculation performed for oligomers noted $\mathrm{Q}_{n}$ in the inset of Figure $2 b$ was such that $m_{X}+m_{Y}=383 \mathrm{Da}$, that is, the expected fully methylated MAA-MMA copolymer with end-group acidic functions being also meth-

Table 1. Weight average molecular weight $\left(M_{\mathrm{w}}\right.$ in $\left.\mathrm{g} \mathrm{mol}^{-1}\right)$ of PMAA standards prior and after methylation calculated from MALDI $\left(M_{\mathrm{w}}{ }^{\mathrm{MALDI}}\right)$ and PGSE $\left(M_{\mathrm{w}}{ }^{\mathrm{PGSE}}\right)$ experiments. SEC data $\left(M_{\mathrm{w}}{ }^{\mathrm{SEC}}\right)$ indicated by the supplier for PMAA standards were used to calculate the values expected for the corresponding methylated PMAA $\left(M_{\mathrm{w}}^{\text {Supplier }}\right)$, knowing the average number of monomer units. The volume of methylation agent $\left(\left(\mathrm{CH}_{3}\right)_{3} \mathrm{SiCHN} \mathrm{N}_{2}\right)$ used for the derivatization of $50 \mathrm{mg}$ of each PMAA standard is also indicated

\begin{tabular}{|c|c|c|c|c|c|c|}
\hline \multirow[b]{2}{*}{ Standard } & \multicolumn{2}{|c|}{ PMAA } & \multirow{2}{*}{$\begin{array}{c}\left(\mathrm{CH}_{3}\right)_{3} \mathrm{SiCHN}{ }_{2} \\
(\mathrm{~mL})\end{array}$} & \multicolumn{3}{|c|}{ Methylated PMAA } \\
\hline & $M_{\mathrm{w}}^{\mathrm{SEC}}$ & $M_{\mathrm{w}}{ }^{\mathrm{MALDI}}$ & & $M_{\mathrm{w}}$ Supplier & $M_{\mathrm{w}}{ }^{\mathrm{MALDI}}$ & $M_{\mathrm{w}}^{\mathrm{PGSE}}$ \\
\hline 1 & 1250 & 1132 & 3.8 & 1445 & 1231 & 1452 \\
\hline 2 & 1700 & 1487 & 4.5 & 1966 & 1634 & 1701 \\
\hline 3 & 3150 & 1951 & 5.6 & 3654 & 2579 & 2928 \\
\hline 4 & 6600 & 4546 & 7.0 & 7664 & 5620 & 6311 \\
\hline
\end{tabular}



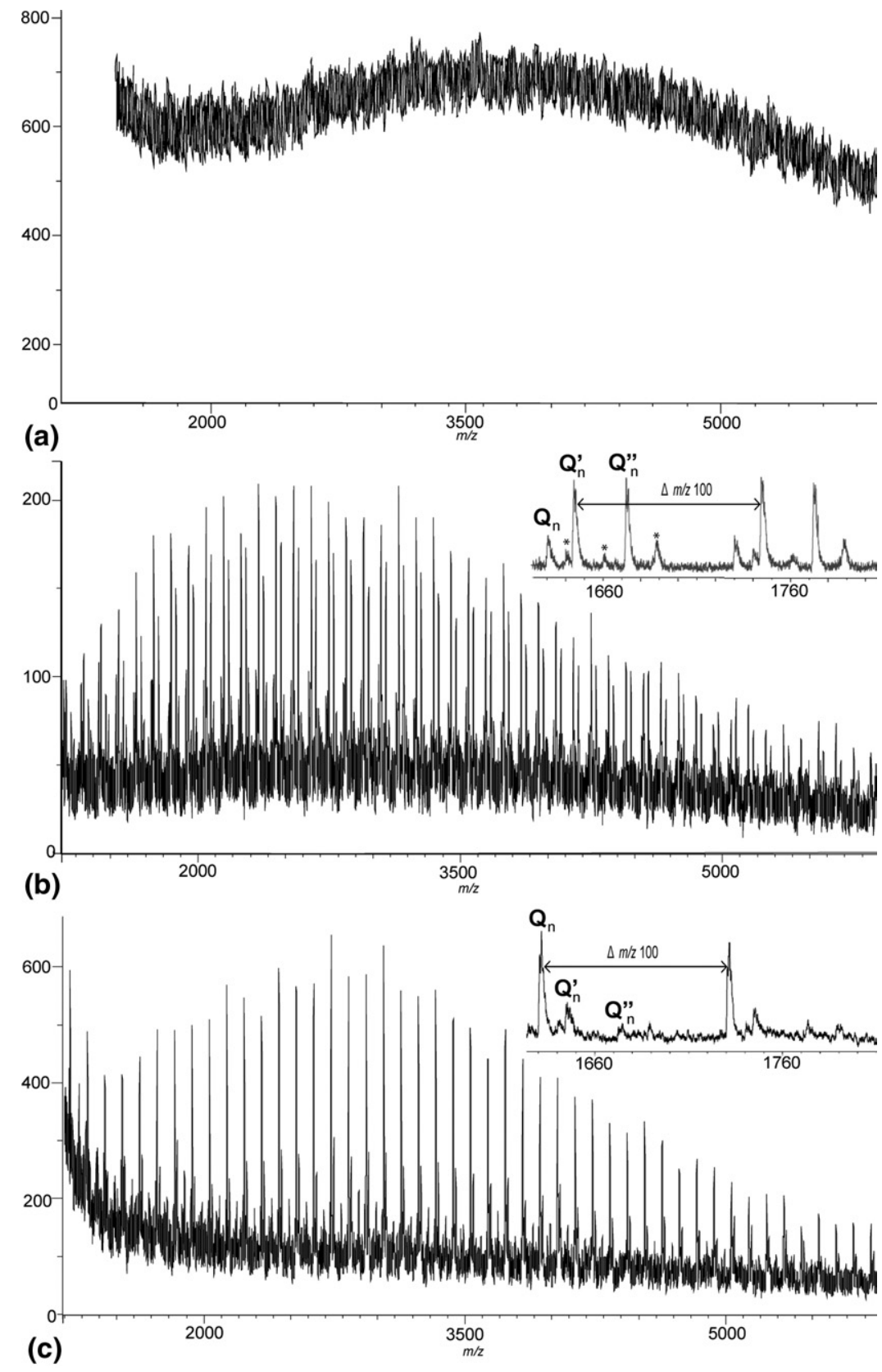

Figure 2. Positive mode MALDI mass spectra of (a) the MAA-MMA (40/60) copolymer prior derivatization, and of the derivatized MAA-MMA (40/60) copolymer using (b) $5.5 \mathrm{~mL}$ and (c) $3.0 \mathrm{~mL}$ of trimethylsilyldiazomethane. THAP was used as the matrix for all experiments, in a matrix-topolymer ratio of 1000:1. In the insets, $\mathrm{Q}_{n}$ refers to the expected PMMA distribution while $\mathrm{Q}^{\prime}{ }_{n}$ and $\mathrm{Q}^{\prime \prime}{ }_{n}$ designate PMMA main by-products. Peaks annotated with a star are minor PMMA by-products.

ylated and the anionic, nonsodiated, sulfonate moiety in the $\mathrm{X}$ end-group. Although these results show that the methylation protocol allowed the studied copolymers to be fully derivatized, the so-formed PMMA oligomers remained of low abundance with regards to ions in distributions $\mathrm{Q}^{\prime}{ }_{n}$ and $\mathrm{Q}^{\prime \prime}{ }_{n}$, as shown in the inset of Figure $2 \mathrm{~b}$. Calculation based on $\mathrm{m} / \mathrm{z}$ data indicated these signals are not accounted for by the presence of partially methylated copolymers in the sample. The use of trimethylsilyldiazomethane as a methylation agent was thus suspected to induce some undesired byproducts due to its high reactivity towards many functional groups [39], in particular an alkene function such as in the $\mathrm{Y}$ end-group of the studied MAA-MMA copolymers (Scheme 1). During methylation of the MAA-MMA (40/60) copolymer I, a $5.5 \mathrm{~mL}$ trimethylsilyldiazomethane volume was necessary to induce a yellow coloration of the reacting medium, indicating the reagent was in excess. Performing the same methylation experiment using a smaller volume of reagent (3 
$\mathrm{mL}$ ) gave rise to the MALDI mass spectrum shown in Figure 2c, where $Q_{n}$ peak abundance was strongly enhanced while by-products $\mathrm{Q}_{n}^{\prime}$ and $\mathrm{Q}^{\prime \prime}{ }_{n}$ became minor distributions, and signal from the three other PMMAlike distributions (annotated with stars in the inset of Figure $2 b$ ) was hardly detected. This result supports the assumption of methylation by-products formed upon reaction with trimethylsilyldiazomethane and the molecule particular end-groups. Structural characterization of these undesired distributions has been addressed using tandem mass spectrometry (vide infra).

Using MALDI and PGSE data, $M_{\mathrm{w}}$ parameters were calculated for $\mathrm{Q}_{n}$ PMMA homopolymers arising from the fully methylated MAA-MMA molecules. Calculation based on MALDI data yielded $M_{\mathrm{w}}$ parameters such as 3915 and 4374 for the methylated copolymers I and II, respectively, while slightly higher values $(+16 \%$ on average) were obtained from PGSE $\left(M_{\mathrm{w}}(\mathrm{I})=4678\right.$ and $\left.M_{\mathrm{w}}(\mathrm{II})=5180\right)$. From these data, a $M_{\mathrm{w}}$ value could then be calculated for each MAA-MMA copolymer since the co-monomer composition and the end-group masses are known from ${ }^{1} \mathrm{H}$ NMR and mass spectrometry experiments, respectively. Indeed, for each methylated copolymer, the $M_{\mathrm{w}}$ value can be expressed as the sum of the end-group masses $\left(\mathrm{m}_{\mathrm{X}}=284\right.$ and $\mathrm{m}_{\mathrm{Y}}=99$, since both terminations were also methylated) and the mass of the polymeric backbone, i.e., $100 n$ with $n$ the average number of MMA units. As the relative proportion of each comonomer, $\mathrm{x}_{\mathrm{MAA}}$ and $\mathrm{x}_{\mathrm{MMA}}$, could be determined by ${ }^{1} \mathrm{H}$ NMR experiments performed on the underivatized samples, a $M_{\mathrm{w}}$ parameter could then calculated for MAA-MMA copolymers as:

$$
M_{\mathrm{W}}=\mathrm{n} \cdot\left(\mathrm{x}_{\mathrm{MAA}} \cdot \mathrm{m}_{\mathrm{MAA}}+\mathrm{x}_{\mathrm{MMA}} \cdot \mathrm{m}_{\mathrm{MMA}}\right)+\mathrm{m}_{\mathrm{X}}+\mathrm{m}_{\mathrm{Y}}
$$

with $m_{X}$ and $m_{Y}$ the mass of the $X$ and $Y$ end-groups prior methylation, respectively. For copolymer I, in which $\mathrm{x}_{\mathrm{MAA}}=0.4$ and $\mathrm{x}_{\mathrm{MMA}}=0.6, M_{\mathrm{w}}$ was found to be 3689 (with $n=35.32$ from MALDI data) and 4409 using PGSE results (with $n=42.95$ ). A similar calculation applied to the 50/50 MAA-MMA copolymer II lead to $M_{\mathrm{w}}$ values of 4067 and 4816 using MALDI and PGSE data, respectively.

Apart from $Q_{n}$ oligomer distribution which resulted from the complete derivatization of random MAAMMA copolymers and hence allowed their $M_{\mathrm{w}}$ parameters to be determined using two independent techniques, two other main distributions $\left(\mathrm{Q}^{\prime}{ }_{n}\right.$ and $\mathrm{Q}^{\prime \prime}{ }_{n}$ in Figure $2 b$ and $c$ ) were evidenced in MALDI, whose abundance could be related to the amount of derivatization reagent added to the copolymer samples. To identify these polymeric by-products produced during methylation of the MAA-MMA molecules, the methylated samples were submitted to ESI to be further dissociated in MS/MS experiments and measured in high-resolution mass spectrometry using an orthogonal acceleration time of flight mass analyzer (oa-TOF). Surprisingly, no signal could be obtained in the positive ESI mode while PMMA-like distributions were readily detected in the negative mode. These oligomers were mainly detected as singly charged molecules in the $600-2000 \mathrm{~m} / \mathrm{z}$ range, indicating a strong bias towards low $\mathrm{m} / \mathrm{z}$ values. The sum of the end-group masses $(97+$ $100 \omega$ for $\mathrm{Q}^{\prime}{ }_{n}$ distribution and $25+100 \omega$ for $\mathrm{Q}^{\prime \prime}{ }_{n}$ distribution, with $\omega$ being an integer) clearly indicated that the same species were detected during ESI and MALDI experiments. The CID behavior of deprotonated oligomers from $\mathrm{Q}_{n}$ distribution was first studied, to be further utilized as a reference to characterize the structure of $\mathrm{Q}^{\prime}{ }_{n}$ and $\mathrm{Q}^{\prime \prime}{ }_{n}$ distribution polymeric by-products.

The ESI-MS/MS spectrum of $\mathbf{Q}_{5}$ detected at $m / z 883.4$ in the negative ion mode is presented in Figure 3. Most product ions could be accounted for by using dissociation rules established by Jackson et al. for PMMA in the

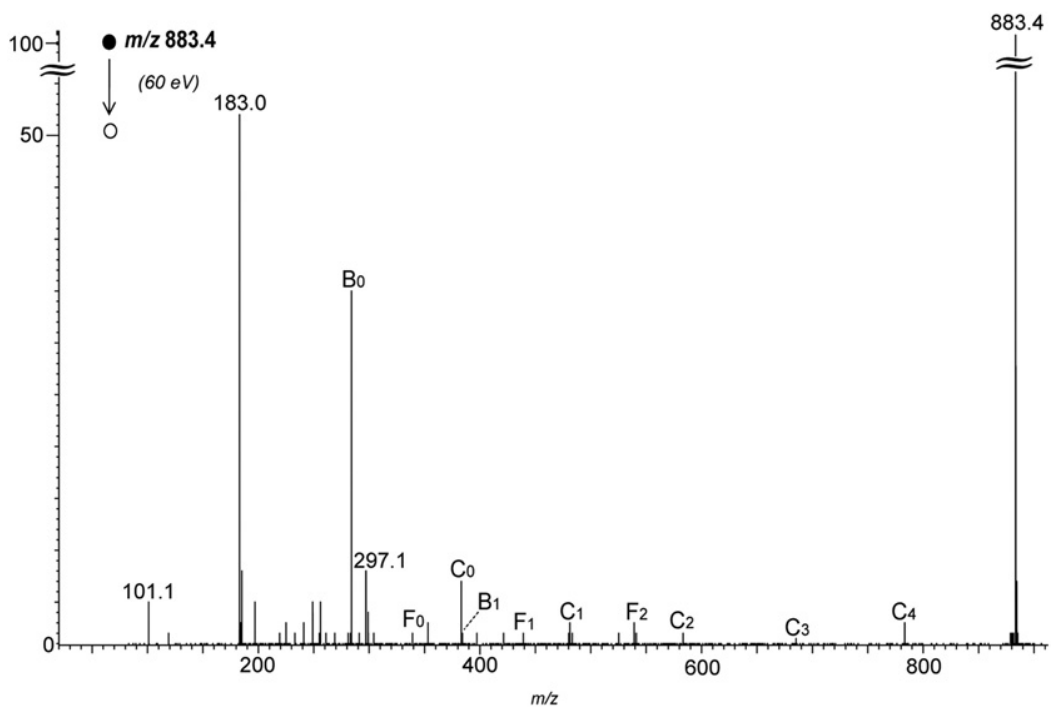

Figure 3. ESI-MS/MS spectrum of $m / z$ 883.4, the anionic homologue of $\mathrm{Q}_{5}$ (collision energy: $60 \mathrm{eV}$, laboratory frame). 
positive ion mode $[40,41]$. Indeed, since the fragmentation routes of cationic PMMA adducts were all proposed to be charge-remote reactions, consisting of direct cleavages of the polymeric backbone and rearrangement reactions, the same mechanisms could be envisaged for the PMMA precursor anion since the negative charge was localized in the sulfonate moiety of the $X$ end-group. As a result, fragment ion series described by Jackson et al. to contain the $\mathrm{Y}$ end-group would not be detected in our case. Indeed, as illustrated in Figure 3, only B, C, and F product ions (as defined by Jackson et al.) were observed (Schemes 1S-3S in Supplementary Material, which can be found in the electronic version of this article). After homolytic cleavage of bonds adjacent to a quaternary carbon in the polymer chain (Scheme $1 S)$, two B fragment ions were detected at $m / z 284.1\left(\mathbf{B}_{0}\right.$, actually the $X$ end-group) and $m / z 384.1\left(B_{1}\right)$, which elemental composition derived from accurate mass measurements, i.e., $\mathrm{C}_{13} \mathrm{H}_{16} \mathrm{O}_{5} \mathrm{~S}^{-}$. $(\mathrm{m} / \mathrm{z}$ 284.0724; error: -13.0 ppm; DBE: 8) and $\mathrm{C}_{18} \mathrm{H}_{24} \mathrm{O}_{7} \mathrm{~S}^{-} \cdot(\mathrm{m} / \mathrm{z}$ 384.1248; error: +16.3 ppm; DBE: 9) respectively, was consistent with the structure of the expected methylated $\mathrm{X}$ endgroup. Two different 1,5-hydrogen rearrangements (Scheme 2S) allowed fragment ions in the $\mathrm{C}$ series to be detected at $m / z 100.1 n+383.1$ (with $n=0-4$, thus indicated the precursor ion at $m / z 883$ was actually a pentamer) while $\mathrm{F}$ product ions (Scheme 3S) were observed at $m / z 100.1 n+339.1$ (with $n=0-2$ ), as supported by accurate mass measurements (See Tables $1 S$ and $2 S$ in Supplementary Material). As previously reported in the CID of electrosprayed PMMA cationic adducts [42], B product ions were of low $\mathrm{m} / \mathrm{z}$ values

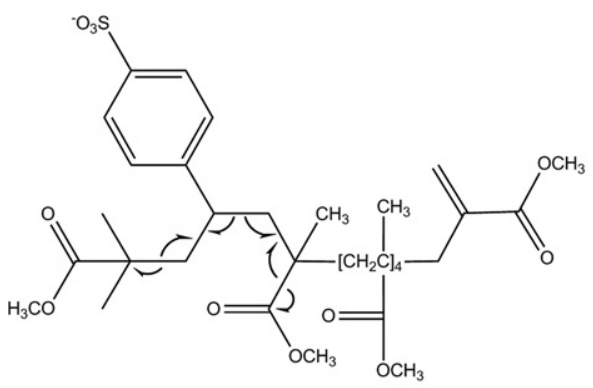

(a)

$m / z 883.4$

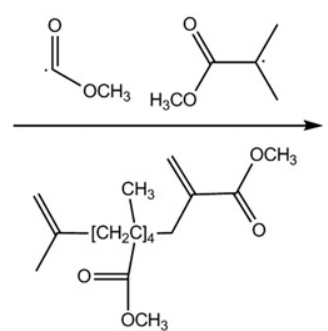<smiles>C=Cc1ccc(OC)cc1</smiles>

$m / z 183.0$

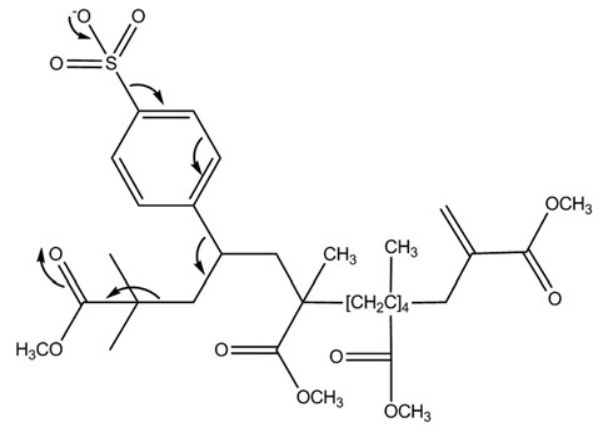

(b) $m / z 883.4$

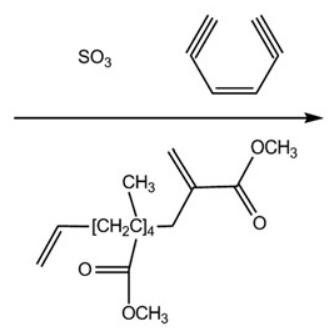<smiles>CC(C)=C(O)O</smiles>

$m / z 101.1$

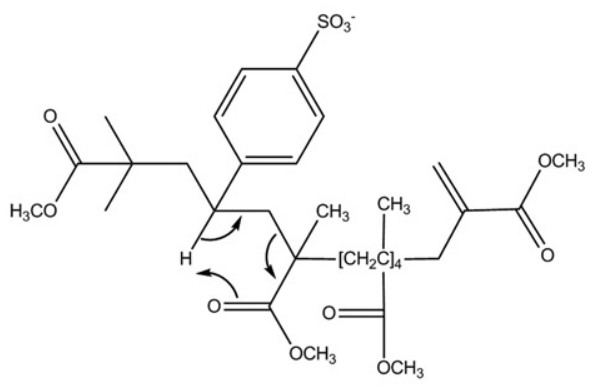

(c)

$m / z 883.4$
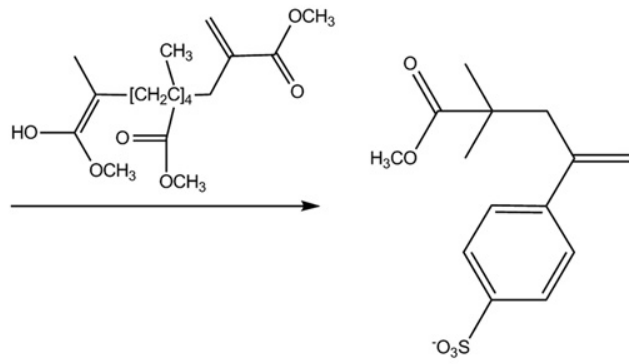

$m / z 297.1$

Scheme 2. Proposed dissociation mechanisms to account for the formation of (a) $\mathrm{m} / \mathrm{z} 183.0$, (b) $\mathrm{m} / \mathrm{z}$ 101.1, and (c) $\mathrm{m} / \mathrm{z} 297.1$ product ions from the $\mathrm{m} / \mathrm{z} 883.4$ precursor ion. 
while $\mathrm{C}$ and $\mathrm{F}$ fragments were detected with low intensity in the higher $\mathrm{m} / \mathrm{z}$ range of the MS/MS spectrum. In addition to these ion series, three peaks could be observed at $m / z$ 297.1, 183.0, and 101.1 in Figure 3. A mechanism consisting of concerted homolytic bond cleavages could be proposed to account for the formation of the intense $\mathrm{m} / \mathrm{z} 183.0$ ion $\left(\mathrm{C}_{8} \mathrm{H}_{7} \mathrm{O}_{3} \mathrm{~S}^{-}\right.$; error: -16.6 ppm; DBE: 7.5) from the $\mathrm{m} / \mathrm{z} 883.4$ precursor ion, as depicted in Scheme 2a. Such a mechanism would also allow $\mathrm{m} / \mathrm{z} 183.0$ to be produced from any $\mathrm{B}, \mathrm{C}$, or $\mathrm{F}$ product ions, thus accounting for its high abundance in the MS/MS spectrum. The $\mathrm{m} / \mathrm{z} 101.1$ and 297.1 product ions would also allow the presence of the expected $X$ end-group to be confirmed. As described in Scheme $\mathbf{2 b}$, $\mathrm{m} / \mathrm{z} 101.1\left(\mathrm{C}_{5} \mathrm{H}_{9} \mathrm{O}_{2}^{-} ; \mathrm{m} / \mathrm{z}\right.$ 101.0608; error: $-4.0 \mathrm{ppm}$; DBE: 1.5$)$ would be produced via a charge-induced mechanism while a 1,5-proton transfer to the carbonyl oxygen atom of the first MMA monomer (Scheme 2c) would account for the formation of $\mathrm{m} / \mathrm{z} 297.1$ $\left(\mathrm{C}_{14} \mathrm{H}_{17} \mathrm{O}_{5} \mathrm{~S}^{-} ; \mathrm{m} / \mathrm{z} 2\right.$ 297.0802; error: +3.0 ppm; DBE: 8.5). It should be added here that none of the reactions typically induced by the presence of MAA units, that is, elimination of water and methanol molecules from MAA/MAA and MAA/MMA pairs, respectively [2931], was observed during CID of $\mathrm{Q}_{n}$ oligomer ions, further confirming all acidic functions in the MAAMMA copolymers have been methylated.

Fragmentation of the two main polymeric by-products generated during methylation of the MAA-MMA was then scrutinized with regards to the dissociation reactions observed for $\mathrm{Q}_{n}$ oligomer ions. MS/MS spectra of any anions produced upon electrospray of oligomers in $\mathrm{Q}^{\prime}{ }_{n}$ distribution showed the $\mathrm{B}, \mathrm{C}$, and $\mathrm{F}$ fragment series (as expected from a PMMA precursor ion), as well as the previously described product ions at $\mathrm{m} / \mathrm{z} 297,183$, and 101, which validate the presence of the same methylated $\mathrm{X}$ end-group as in $\mathrm{Q}_{n}$. As a result, according to the oligomer $\mathrm{m} / \mathrm{z}$ values and the mass of methylated $\mathrm{X}$ end-group (284 Da), the $\mathrm{Y}$ end group mass was found to be such as $m_{Y}=13+100 \omega$, with $\omega$ an integer number. The case where $\omega=2$ could correspond to a $\mathrm{Y}$ end-group with the structure proposed in Scheme 3, which would arise from a Michaël addition of trimethylsilyldiazomethane, starting with the nucleophilic addition of diazomethanide to the $\alpha, \beta$-unsaturated carbonyl moiety. This structural assumption was supported by accurate mass measurements of oligomers in the MS spectrum, using two $Q_{n}$ oligomer ions as internal standards to bracket the targeted analytes (Table 2). Using the same methodology, MS/MS data together with accurate mass measurements (Table 2) allowed us to propose the structure depicted in Scheme 3 for $\mathrm{Q}^{\prime \prime}{ }_{n}$ PMMA anions, as the result of a reaction previously reported to occur between diazomethane and alkenes [39]. As for $\mathrm{Q}_{n}$ oligomer ions, all product ions generated upon CID of the oligomer by-products contained the ionized X end group and no detailed information could be obtained from MS/MS data about the structure of the $\mathrm{Y}$ end
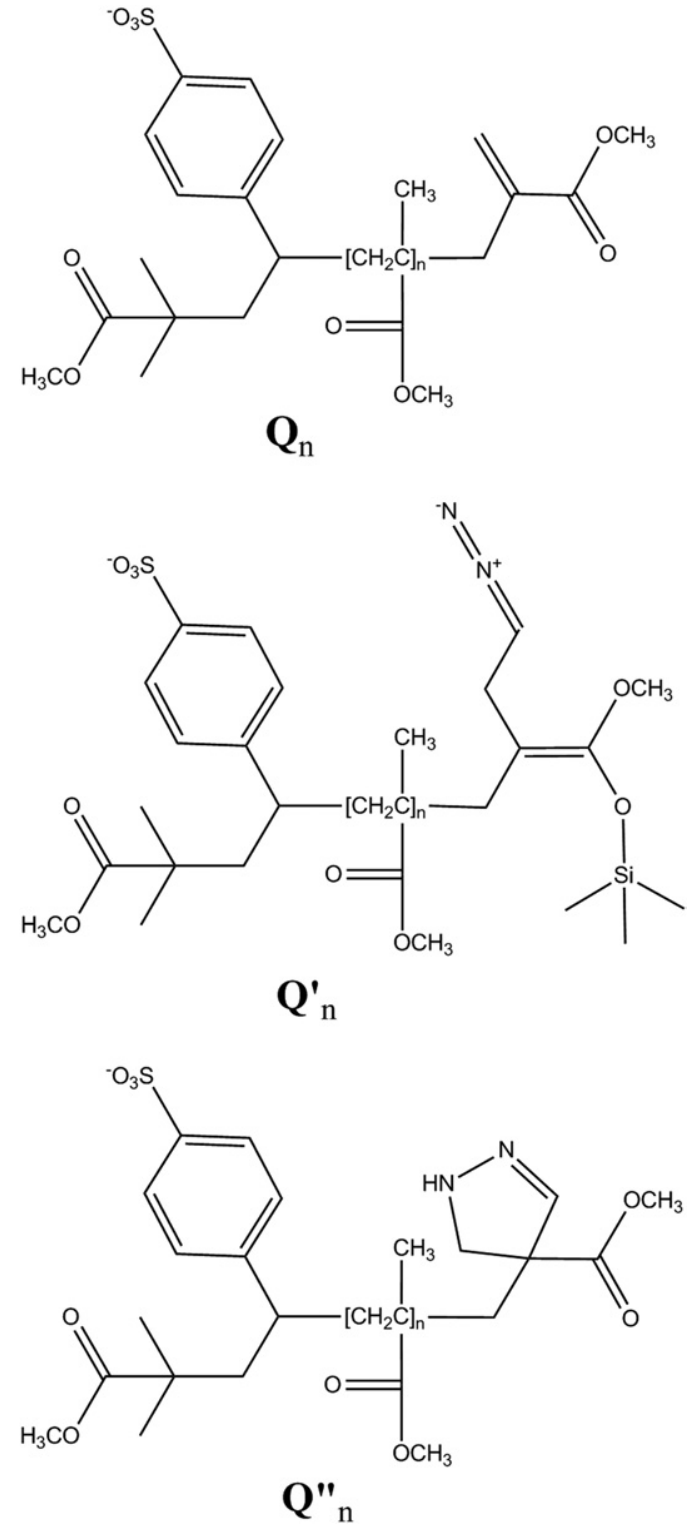

Scheme 3. Structures proposed for $\mathrm{Q}_{n}, \mathrm{Q}^{\prime}{ }_{n}$ and $\mathrm{Q}^{\prime \prime}{ }_{n}$ PMMA polymeric species.

group. Nevertheless, $\mathrm{Q}^{\prime}{ }_{n}$ and $\mathrm{Q}^{\prime \prime}{ }_{n}$ distributions could reasonably be attributed to by-products due to an excess of trimethylsilyldiazomethane since their formation could readily be minimized by decreasing the quantity of methylation agent in the reaction medium, as previously shown in Figure 2c.

\section{Conclusion}

The derivatization strategy used in this study was shown to allow the $M_{\mathrm{w}}$ parameter to be determined for amphiphilic random copolymers of MAA-MMA that can be quantitatively derivatized to yield homopolymers, for which this information can directly be obtained from the MALDI mass spectrum. Since comonomeric composition was assessed by ${ }^{1} \mathrm{H}$ NMR, 
Table 2. Composition and accurate mass measurements of some $\mathrm{Q}_{n}^{\prime}$ and $\mathrm{Q}_{n}{ }_{n}$ PMMA by-products produced in negative mode electrospray ionization

\begin{tabular}{|c|c|c|c|c|c|}
\hline$\underline{n}$ & Elemental composition & $(\mathrm{m} / \mathrm{z})_{\text {theo }}$ & $(m / z)_{\exp }$ & Error (ppm) & DBE \\
\hline \multicolumn{6}{|c|}{$\mathrm{Q}_{n}^{\prime}$ by-products } \\
\hline 2 & $\mathrm{C}_{32} \mathrm{H}_{49} \mathrm{~N}_{2} \mathrm{O}_{11} \mathrm{SSi}^{-}$ & 697.2831 & 697.2889 & +8.2 & 12.5 \\
\hline 3 & $\mathrm{C}_{37} \mathrm{H}_{57} \mathrm{~N}_{2} \mathrm{O}_{13} \mathrm{SSi}^{-}$ & 797.3356 & 797.3402 & +5.8 & 13.5 \\
\hline 4 & $\mathrm{C}_{42} \mathrm{H}_{65} \mathrm{~N}_{2} \mathrm{O}_{15} \mathrm{SSi}^{-}$ & 897.3880 & 897.3932 & +5.8 & 14.5 \\
\hline 5 & $\mathrm{C}_{47} \mathrm{H}_{73} \mathrm{~N}_{2} \mathrm{O}_{17} \mathrm{SSi}^{-}$ & 997.4404 & 997.4469 & +6.5 & 15.5 \\
\hline 6 & $\mathrm{C}_{52} \mathrm{H}_{81} \mathrm{~N}_{2} \mathrm{O}_{19} \mathrm{SSi}^{-}$ & 1097.4929 & 1097.4936 & +0.6 & 16.5 \\
\hline \multicolumn{6}{|c|}{$\mathrm{Q}^{\prime \prime}{ }_{n}$ by-products } \\
\hline 3 & $\mathrm{C}_{34} \mathrm{H}_{49} \mathrm{~N}_{2} \mathrm{O}_{13} \mathrm{~S}^{-}$ & 725.2960 & 725.2948 & -1.7 & 13.5 \\
\hline 4 & $\mathrm{C}_{39} \mathrm{H}_{57} \mathrm{~N}_{2} \mathrm{O}_{15} \mathrm{~S}^{-}$ & 825.3485 & 825.3471 & -1.7 & 14.5 \\
\hline 5 & $\mathrm{C}_{44} \mathrm{H}_{65} \mathrm{~N}_{2} \mathrm{O}_{17} \mathrm{~S}^{-}$ & 925.4009 & 925.4017 & +0.9 & 15.5 \\
\hline 6 & $\mathrm{C}_{49} \mathrm{H}_{73} \mathrm{~N}_{2} \mathrm{O}_{19} \mathrm{~S}^{-}$ & 1025.4533 & 1025.4515 & -1.8 & 16.5 \\
\hline 7 & $\mathrm{C}_{54} \mathrm{H}_{81} \mathrm{~N}_{2} \mathrm{O}_{21} \mathrm{~S}^{-}$ & 1125.5058 & 1125.5020 & -3.4 & 17.5 \\
\hline
\end{tabular}

there is no information loss whatsoever. This procedure does indeed require sample manipulation, but the proposed chemical derivatization is relatively fast and simple to implement. Moreover, up to now, this is the only available method for providing the analyst with the copolymer molecular weight. This approach is currently extended in our laboratory to reduce the amphiphilic character of other MAA-based amphiphilic macromolecules so that both their composition and $M_{\mathrm{w}}$ parameter could be determined in a single MALDI experiment.

\section{Acknowledgments}

The authors thank Arkema for their financial support. R.G. thanks Arkema for their 3-y grant. L.C. and S.V. acknowledge support from Spectropole, the Analytical Facility of Aix-Marseille University, by allowing a special access to the instruments purchased with European funding (FEDER OBJ2142-3341).

\section{Appendix A Supplementary Material}

Supplementary material associated with this article may be found in the online version at doi:10.1016/ j.jasms.2010.02.007.

\section{References}

1. Forster, S.; Abetz, V.; Muller, A. H. E. Polyelectrolyte Block Copolymer Micelles. In Polyelectrolytes with Defined Molecular Architecture II; Lavoisier: Paris, France, 2004; Vol. CLXVI, pp 173-210.

2. Mun, G. A.; Nurkeeva, Z. S.; Irmukhametova, G. S.; Guven, O. Synthesis, Characterization, and Adsorption Study of the Uranyl Ions by Hydrogels Based on Polyethylene Glycol and Methacrylic Acid Copolymers. Nucl. Instrum. Methods Phys. Res. Sect. B 2007, 265, 379-384.

3. Colombani, O.; Ruppel, M.; Burkhardt, M.; Drechsler, M.; Schumacher, M.; Gradzielski, M.; Schweins, R.; Muller, A. H. E. Structure of Micelles of Poly(n-Butyl Acrylate)-Block-Poly (Acrylic Acid) Diblock Copolymers in Aqueous Solution. Macromolecules 2007, 40, 4351-4362.

4. Colombani, O.; Ruppel, M.; Schubert, F.; Zettl, H.; Pergushov, D. V.; Muller, A. H. E. Synthesis of Poly(n-Butyl Acrylate)-Block-Poly(Acrylic Acid) Diblock Copolymers by ATRP and Their Micellization in Water. Macromolecules 2007, 40, 4338-4350.

5. Burkhardt, M.; Martinez-Castro, N.; Tea, S.; Drechsler, M.; Babin, I.; Grishagin, I.; Schweins, R.; Pergushov, D. V.; Gradzielski, M.; Zezin, A. B.; Muller, A. H. E. Polyisobutylene-Block-Poly(Methacrylic Acid) Diblock Copolymers: Self-Assembly in Aqueous Media. Langmuir 2007, 23, 12864-12874.
6. Pergushov, D. V.; Remizova, E. V.; Feldthusen, J.; Zezin, A. B.; Muller, A. H. E.; Kabanov, V. A. Novel Water-Soluble Micellar Interpolyelectrolyte Complexes. J. Phys. Chem. B 2003, 107, 8093-8096.

7. Ravi, P.; Wang, C.; Tam, K. C.; Gan, L. H. Association Behavior of Poly(Methacrylic Acid)-Block-Poly(Methyl Methacrylate) in Aqueous Medium: Potentiometric and Laser Light Scattering Studies. Macromolecules 2003, 36, 173-179.

8. Yao, H.; Ravi, P.; Tam, K. C.; Gan, L. H. Association Behavior of Poly(Methyl Methacrylate-b-Methacrylic Acid-b-Methyl Methacrylate) in Aqueous Medium. Polymer 2004, 45, 2781-2791.

9. Yao, J.; Ravi, P.; Tam, K. C.; Gan, L. H. Association Behavior of Poly(Methyl Methacrylate-Block-Methacrylic Acid) in Aqueous Medium. Langmuir 2004, 20, 2157-2163.

10. Adhikari, R.; Michler, G. H. Influence of Molecular Architecture on Morphology and Micromechanical Behavior of Styrene/Butadiene Bblock Copolymer Systems. Prog. Polym. Sci. 2004, 29, 949-986.

11. Gotzamanis, G.; Tsitsilianis, C. Stimuli-Responsive A-b-(B-co-C) Diblock Terpolymers Bearing Polyampholyte Sequences. Macromol. Rapid Commun. 2006, 27, 1757-1763.

12. Gotzamanis, G.; Tsitsilianis, C. Design of Responsive DoubleHydrophilic A-b-(B-co-C) Diblock Terpolymers with Tunable Thermosensitivity. Polymer 2007, 48, 6226-6233.

13. Zielinski, J. M.; Spontak, R. J. Thermodynamic Considerations of Triblock Copolymers with a Random Middle Block. Macromolecules.1992, 25, 5957-5964.

14. Laurer, J. H.; Smith, S. D.; Samseth, J.; Mortensen, K.; Spontak, R. J. Interfacial Modification as a Route to Novel Bilayered Morphologies in Binary Block Copolymer/Homopolymer Blends. Macromolecules 1998, 31, 4975-4985.

15. Grubisic, Z.; Rempp, P.; Benoit, H. A Universal Calibration for Gel Permeation Chromatography. J. Polym. Sci. B Polym. Phys. 1996, 34, 1707-1713.

16. Mori, S.; Barth, H. G. Size Exclusion Chromatography; Springer-Verlag: Berlin, Germany, 1999; pp 185-198.

17. Medrano, R.; Laguna, M. T. R.; Saiz, E.; Tarazona, M. P. Analysis of Copolymers of Styrene and Methyl Methacrylate Using Size Exclusion Chromatography with Multiple Detection. Phys. Chem., Chem. Phys. 2003, 5, 151-157.

18. Stejskal, E. O.; Tanner, J. E. Spin Diffusion Measurements: Spin Echoes in the Presence of a Time-Dependent Field Gradient. J. Chem. Phys. 1965, 42, 288-291.

19. Stilbs, P. Fourier Transform Pulsed-Gradient Spin-Echo Studies of Molecular Diffusion. Prog. Nucl. Magn. Reson. Spectrosc. 1987, 19, 1-45.

20. Price, W. S. Pulsed-Field Gradient Nuclear Magnetic Resonance as a Tool for Studying Translational Diffusion: Part 1. Basic Theory. Concepts Magn. Reson. 1997, 9, 299-336.

21. Chen, A.; Wu, D.; Johnson, C. S. Jr. Determination of Molecular Weight Distributions for Polymers by Diffusion Ordered NMR. J. Am. Chem Soc. 1995, 117, 7965-7970.

22. Håkansson, B.; Nydén, M.; Söderman, O. The Influence of Polymer Molecular Weight Distributions on Pulsed Field Gradient Nuclear Magnetic Resonance Self-Diffusion Experiments. Colloid. Polym. Sci. 1999, 278, 399-405

23. Viel, S.; Capitani, D.; Mannina, L.; Segre, A. Diffusion-Ordered NMR Spectroscopy: A Versatile Tool for the Molecular Weight Determination of Uncharged Polysaccharides. Biomacromolecules 2003, 4, 1843-1847.

24. Mazarin, M.; Viel, S.; Allard-Breton, B.; Thevand, A.; Charles, L. Use of Pulsed Gradient Spin-Echo NMR as a Tool in MALDI Method Development for Polymer Molecular Weight Determination. Anal. Chem. 2006, 78, 2758-2764.

25. Barrere, C.; Mazarin, M.; Giordanengo, R.; Phan, T. N. T.; Thevand, A.; Viel, S.; Charles, L. Molecular Weight Determination of Block Copolymers by Pulsed Gradient Spin Echo NMR. Anal. Chem. 2009, 81, 8054-8060 
26. Montaudo, G.; Lattimer, R. P. Mass Spectrometry of Polymers; CRC Press: Boca Raton, 2002; pp 523-552.

27. Pasch, H.; Schrepp, W. MALDI-TOF Mass Spectrometry of Synthetic Polymers; Springer-Verlag: Berlin, Germany, 2002; pp 85-106.

28. Leenheer, J. A.; Rostad, C. E.; Gates, P. M.; Furlong, E. T.; Ferrer, I. Molecular Resolution and Fragmentation of Fulvic Acid by Electrospray Ionization/Multistage Tandem Mass Spectrometry. Anal. Chem. 2001, 73, 1461-1471.

29. Giordanengo, R.; Viel, S.; Allard-Breton, B.; Thevand, A.; Charles, L. Tandem Mass Spectrometry of Poly(Methacrylic Acid) Oligomers Produced by Negative Mode Electrospray Ionization. J. Am. Soc. Mass Spectrom. 2009, 20, 25-33.

30. Giordanengo, R.; Viel, S.; Allard-Breton, B.; Thevand, A.; Charles, L. Positive Mode Electrospray Tandem Mass Spectrometry of Poly(Methacrylic Acid) Oligomers. Rapid Commun. Mass Spectrom. 2009, 23, 1557-1562.

31. Giordanengo, R.; Viel, S.; Hidalgo, M.; Allard-Breton, B.; Thevand, A.; Charles, L. Structural Characterization of a Poly(Methacrylic Acid)Poly(Methyl Methacrylate) Copolymer by Nuclear Magnetic Resonance and Mass Spectrometry. Anal. Chim. Acta 2009, 654, 49-58.

32. Wyatt, M. F.; Schaeffer, N.; Tan, B.; Cooper, A. I. Strategies for the Analysis of Poly(Methacrylic Acid) by Matrix-Assisted Laser Desorption/Ionization Time-of-Flight Mass Spectrometry. J. Am. Soc. Mass Spectrom. 2007, 18, 1507-1510.

33. Girod, M.; Mazarin, M.; Phan, T. N. T.; Gigmes, D.; Charles, L. Determination of Block Size in Poly(Ethylene Oxide)-b-Polystyrene Block Copolymers by Matrix-Assisted Laser Desorption/Ionization Time-of-Flight Mass Spectrometry. J. Polym. Sci. A Polym. Chem. 2009, $47,3380-3390$
34. Couvreur, L.; Lefay, C.; Belleney, J.; Charleux, B.; Guerret, O.; Magnet, S. First Nitroxide-Mediated Controlled Free-Radical Polymerization of Acrylic Acid. Macromolecules 2003, 36, 8260-8267.

35. Arakawa, R.; Egami, S.; Okuno, S. An effective Derivatization Method for Matrix-Assisted Laser Desorption/Ionization Mass Spectrometry of Poly(Acrylic Acid). J. Mass Spectrom. 2006, 41, 549-550.

36. Charles, L. Influence of Internal Standard Charge State on the Accuracy of Mass Measurements in Orthogonal Acceleration Time-of-Flight Mass Spectrometers. Rapid Commun. Mass Spectrom. 2008, 22, 151-155.

37. Edwards, S. F.; Doi, M. The Theory of Polymer Dynamics. Oxford University Press: New York, 1986; p. 391.

38. Danis, P. O; Karr, D. E. Analysis of Poly(Styrenesulfonic Acid) by Matrix-Assisted Laser-Desorption Ionization Time-of-Flight MassSpectrometry. Macromolecules 1995, 28, 8548-8551.

39. Mori, S.; Sakai, I.; Aoyama, T.; Shioiri, T. New Methods and Reagents in Organic-Synthesis. 28. A Convenient and Efficient Preparation of Trimethylsilyldiazomethane (Tmschn2) Using Diphenyl Phosphorazidate (DPPA). Chem. Pharm. Bull. 1982, 30, 3380-3382.

40. Jackson, A. T.; Yates, H. T.; Scrivens, J. H.; Critchley, G.; Brown, J.; Green, M. R.; Bateman, R. H. The Application of Matrix-Assisted Laser Desorption/Ionization Combined with Collision-Induced Dissociation to the Analysis of Synthetic Polymers. Rapid Commun. Mass Spectrom. 1996, 10, 1668-1674.

41. Jackson, A. T.; Yates, H. T.; Scrivens, J. H.; Green, M. R.; Bateman, R. H. Utilizing Matrix-Assisted Laser Desorption/Ionization-Collision Induced Dissociation for the Generation of Structural Information from Poly(Alkyl Methacrylate)s. I. Am. Soc. Mass Spectrom. 1997, 8, 1206-1213.

42. Jackson, A. T.; Slade, S. E.; Scrivens, J. H. Characterization of Poly(Alkyl Methacrylate)s by Means of Electrospray Ionization-Tandem Mass Spectrometry (ESI-MS/MS). Int. J. Mass Spectrom. 2004, 238, 265-277. 